\title{
El sufijo «infinitivizador» en quechua ${ }^{1}$
}

\author{
Rolando Rocha MartínEZ \\ Universidad Nacional Mayor de San Marcos \\ rolandop7@hotmail.com
}

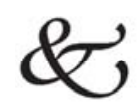

\section{Resumen}

El quechua presenta un sufijo formador de nuevas palabras que ha sido descrito como derivativo nominal deverbativo, es decir, como un sufijo que deriva nombres a partir de elementos lexicales de naturaleza verbal. Sin embargo, la categorización de este sufijo como un derivativo nominal trae consigo una serie de escollos de los que daremos cuenta en el presente estudio con la finalidad de plantear una propuesta factible respecto del estatus nominal o verbal del morfema involucrado. Nuestra investigación propone una recategorización del sufijo en cuestión ya que las descripciones taxonómicas solamente lo han definido como una terminación propiamente infinitiva que constituye formas no personales, pero cuya naturaleza es de tipo nominal. El problema potencial que encontramos en el análisis tradicional de este sufijo es que nos remite a una forma nominal como producto derivado a partir de un verbo; no obstante, el comportamiento de dicha forma derivada dista mucho de ser propiamente un nombre.

Palabras claves: Quechua, morfología derivativa, sufijo infinitivizador.

\begin{abstract}
The Quechua has a suffix that forms new words which are been described as nominal deverbativo derivative, ie as a suffix that derived names from lexical items of a verbal nature. However, the categorization of

1 Este artículo es el producto parcial de la investigación «Sistema derivativo del quechua de Aurahuá-Chupamarca», dirigida por la Dra. Isabel Gálvez Astorayme el año 2008, y en la que coparticipé en calidad de miembro colaborador. Se presentó una versión preliminar en el I Coloquio sobre Lengua y Cultura Andina organizado por la Cátedra de Lengua Quechua de la UNMSM el año 2009, y una versión ampliada con datos actuales de la variedad de Huánuco (Huamalíes) en el Congreso de Linguiística y Educación «Alberto Escobar Zambrano» el año 2010. Agradezco el importante aporte de Franklin Espinoza Bustamante, en lo que concierne a la contrastación de datos de la variedad de Huánuco, el cual ha sido sustancial.
\end{abstract}

LETRAS 81 (116), 2010 
this suffix as a nominal derivative entails a number of pitfalls which we try to solve in the present study in order to propose a feasible proposal with respect to the nominal or verbal status of the morpheme involved. Our research proposes a reclassification of the suffix in question, as the only taxonomic descriptions have defined it as a termination infinitive which constitutes personal forms, but whose nature is the nominal rate. The potential problem found in the traditional analysis of this suffix is refers to a nominal form as a product derived from a verb, however, the behavior of the derivative form is far from being strictly a name

Key words: Quechua, derivative morphorlogy, suffix infinitivizador.

\section{Introducción}

La literatura sobre los diversos dialectos quechuas es profusa. La labor investigativa sobre sus características gramaticales, entendidas como un mosaico complejo y no como una lengua homogénea, ha sido proficua. Los trabajos sobre el quechua, iniciados por Alfredo Torero el año 1964 con la obra intitulada "Los dialectos quechuas", la cual define el rumbo de la andinística peruana, constituyen el punto de partida de numerosas descripciones sobre los subgrupos dialectales del quechua. La naturaleza de los complejos dialectales que conforman esta familia linguiística es aglutinante, específicamente sufijante, lo cual supone que a una forma base se le agregan morfemas con propiedades flexivas, derivativas y hasta discursivas. La presente investigación aborda el problema de la categorización de un sufijo, tradicionalmente clasificado como derivativo nominal, con la finalidad de redefinir la naturaleza del sufijo en cuestión. Nuestra indagación procura dilucidar las propiedades derivativas del sufijo en busca de una explicación plausible acerca del producto derivado final y las consecuencias de este en la clasificación del morfema. A manera de introducción pasaremos a definir los aspectos más relevantes de los procesos derivativos en quechua, los cuales, en rigor, se clasifican en dos clases: derivación nominal y derivación verbal.

\section{La derivación en quechua}

Los dialectos quechuas ofrecen un espectro morfemático complejo, en el que ciertas marcas morfológicas son portadoras de información de tipo gramatical (caso, persona poseedora, actora, etc.), mientras que otras modifican la estructura léxica de las palabras generando nuevo vocabulario; estas últimas están involucradas en la derivación; a saber, la generación de palabras a partir de formas bases que devienen, al añadirse un morfema, en una nueva palabra. La derivación como mecanismos morfológico genera léxico nuevo mante- 
niendo las propiedades léxicas de la forma base o modificando léxicamente la forma base. Esta puede ser de dos tipos: nominal y verbal, de acuerdo con el elemento final generado. Asimismo, la derivación nominal puede ser de tipo intracategorial o denominativa, y transcategorial o deverbativa (Torero 2002: 62). Se dice que la derivación de tipo nominal es denominativa cuando los nombres son formados a partir de nombres. Tal es el caso del sufijo $\{$-yuq\}, que modifica nombres para devenir en nombres. Veamos el siguiente ejemplo:

(i) Derivación de nombre a nombre

$$
\begin{aligned}
& \text { /čukča/ 'pelo' } \\
& \text { /čukča-yuq/ 'el que tiene pelo' } \\
& \text { /qu } \lambda \mathrm{qi} / \quad \text { 'dinero' } \\
& \text { /qu^qi-yuq/ 'quien tiene dinero' }
\end{aligned}
$$

En los casos anteriores notamos que la naturaleza categorial de los elementos nominales no se modifica; esto es, el nombre "pelo» deviene en un nuevo nombre que se parafrasea como "el que tiene o es dueño del pelo». Por otro lado, la derivación de tipo nominal es deverbativa, cuando la forma base del nombre derivado es un verbo, lo cual implica que la forma base modifique sus rasgos categoriales: en este caso un verbo deja de ser un nombre para volverse verbo. Tal proceso es evidenciado en el siguiente caso:

(ii) Derivación de verbo a nombre

$$
\begin{array}{ll}
\text { /puJu-/ } & \text { 'dormir' } \\
\text { /puJu-na/ } & \text { 'lugar para dormir' }
\end{array}
$$

La derivación verbal también sigue el mismo derrotero y puede clasificarse de dos formas: intracategorial o deverbativa o transcategorial denominativa. La diferencia es la siguiente: mientras que la verbalización deverbativa (entendida la verbalización como un proceso derivativo que involucra la consecución de un nuevo verbo) genera verbos a partir de verbos, mientras que la verbalización denominativa hace lo propio pero a partir de nombres. Veamos algunos casos.

(iii) Derivación de verbo a verbo

$\begin{array}{ll}\text { /ranti-/ } & \text { 'comprar' } \\ \text { /ranti-či/ } & \text { 'hacer comprar' } \\ \text { /puJu-/ } & \text { 'dormir' } \\ \text { /puJu- či/ } & \text { 'hacer dormir' }\end{array}$


(iv) Derivación de nombre a verbo (Torero 2002: 62)

$$
\begin{array}{ll}
\text { /puka-/ } & \text { 'rojo' } \\
\text { /puka-ya/ } & \text { 'enrojecer' } \\
\text { /wasi-/ } & \text { 'casa' } \\
\text { /wasi-ča/ } & \text { 'hacer, construir una casa' }
\end{array}
$$

\section{El sufijo «infinitivizador» $\{-\mathrm{y}\}$}

El sufijo que ocupará nuestra reflexión en lo que sigue ha sido tradicionalmente categorizado como sufijo derivativo nominal deverbativo. Siguiendo la clasificación simple ofrecida líneas arriba, nos percatamos que este sufijo modifica verbos para volverlos nombres; esto es, el producto derivado a partir del sufijo involucrado cambia completamente su naturaleza verbal para dar paso a una categoría léxica nueva.

La literatura sobre las variedades quechuas le confieren estatus nominal. El primero en conferirle dicho estatus en el marco de las descripciones sistemáticas del quechua es Parker (1965: 58) quien asume que «/-y/ deriva un nombre Na que se refiere a una acción en abstracto, sin limitación espacial». Esta definición ha sido seguida por diversos autores. Así tenemos las siguientes definiciones del sufijo:

(1) «\{-Y\} deriva un nombre que alude a la acción en abstracto» (Escribens, A. y Paul Proulx 1970: 47).

(2) «-y, infinitivo. Es ambivalente por cuanto funciona también como marcador del imperativo. Este sufijo deriva un nombre que alude a una acción en abstracto; es decir, sin referencia temporal, modal ni aspectual. Lo que quiere decir es que el sufijo -y en función de infinitivo forma nombres abstractos» (Quesada 1976: 100).

(3) «El infinitivo -y. Añadido a una raíz tema verbal este sufijo hace que la acción adquiera un matiz abstracto, desprovisto de toda referencia temporal o aspectual» (Cerrón-Palomino 1976: 160).

(4) «[...] (a) -y 'infinitivizador' [...] deriva temas que expresan la acción verbal en abstracto (los llamados infinitivos)» (Cerrón-Palomino 1987: 272).

(5) «-y. Indica el infinitivo. Es decir, deriva un sustantivo abstracto que no tiene referencia personal: Pukllay, "jugar el juego". Piluta pukllay, "jugar pelota, juego de pelota (fútbol)» (Clodoaldo Soto 2006: 244).

La cita de Quesada es reveladora, ya que deja evidencia del carácter ambivalente del sufijo en la cita (2). Salvo en la cita (1), las demás, de manera coincidente, definen al morfema como infinitivo, y, de hecho, la misma denominación trae ciertos inconvenientes, ya que lo infinitivo es simplemente una forma no personal, y eso no necesariamente supone que la forma verbal base modifique sus propiedades categoriales. Dejaremos este punto para la 
discusión posterior en este artículo. A continuación, definiremos la cuestión para, posteriormente, analizar las implicancias del sufijo en la presunta modificación de verbos.

\section{La paradoja entre formas nominales y la asignación de caso acu- sativo}

Un problema con la clasificación del sufijo -y como nominalizador es que al ser el origen de un cambio categorial, la raíz nueva carecería de propiedades verbales; esto trae una serie de implicancias en la asignación de caso, por ejemplo, tal y como veremos en el capítulo siguiente. Veamos a continuación, algunas de las incidencias de las formas "derivadas» en construcciones oracionales.

$$
\begin{aligned}
& \text { Nuqa tanta -ta ranti -y -ta muna: } \\
& \text { Yo pan AC. comprar INF AC. querer 1PA } \\
& \text { 'yo quiero comprar pan’ }
\end{aligned}
$$

Notamos en el caso anterior que la forma, el sufijo $-y$, agregado a la forma verbal ranti- 'comprar', define un argumento (tanta 'pan'), el cual porta la marca acusativa - $t a$. Visto así, el verbo ranti- asigna caso acusativo y, en términos teóricos, el caso acusativo solamente puede ser asignado por un verbo (Fernández y Anula 1995: 167). Sin embargo, siguiendo sensu stricto la clasificación y característica del sufijo -y, el verbo ranti- debería haber cambiado de categoría gramatical (notemos que en la oración ranti- porta la marca morfológica derivativa en cuestión). De esta paradoja, desprendemos las siguientes interrogantes:

a) ¿El sufijo derivativo $\{-y\}$ realmente determina el cambio categorial de las formas verbales a las que se añade?

b) ¿El sufijo «infinitivizador» es un morfema derivativo de tipo nominal?

c) ¿Qué evidencia gramatical existe para sostener que el sufijo $\{-\mathrm{y}\}$ recategoriza verbos?

Nuestra hipótesis es que el sufijo $\{-y\}$ es una marca morfológica que define verbos en forma impersonal. Las formas verbales impersonales no requieren modificar sus características categoriales. Desde ese punto de vista, el verbo no necesariamente deriva nombres a partir de verbos, puesto que un verbo en su forma impersonal puede cumplir, sintácticamente, muchas de las funciones de un nombre. En la variedad quechua de Aurahuá, por ejemplo, la marca infinitiva está siendo reemplazada por la forma infinitiva castellana. Esta evidencia supone que los hablantes de esta variedad quechua optan por la marca que define formas verbales impersonales en castellano, probablemente porque la función que cumple es similar en quechua. Un indicio de que nuestra 
propuesta es adecuada es la polifuncionalidad del sufijo quechua -y. Además, la adopción de la marca infinitiva castellana en formas verbales quechuas evidencia un recurso para uniformizar la función en cuestión. La propuesta teórica que asumiremos para clarificar la naturaleza del sufijo es la TPP (Teoría de Principios y Parámetros) y evaluaremos las diversas descripciones del quechua a fin de contrastarlas. Adicionalmente presentaremos evidencia actual del comportamiento de este sufijo en algunos dialectos quechuas. El resultado concreto de nuestra evaluación supone que las formas verbales no cambian su naturaleza categorial, sino que al ser formas no personales son pasibles de cumplir funciones nominales.

\section{Léxico quechua y rasgos categoriales ${ }^{2}$}

El diseño gramatical en el enfoque generativo de Principios y Parámetros define al léxico como un nivel independiente en el que las unidades que lo conforman cuentan con especificaciones de distinto tipo: rasgos categoriales, rasgos gramaticales, un marco de subcategorización, etc. Los rasgos categoriales conforman para de la información sintáctica que las unidades léxicas poseen y definen una matriz básica de unidades a partir de rasgos de tipo nominal y verbal, los cuales están motivados por la ocurrencia o no de elementos después de otros en una construcción sintáctica. De esta manera, si los elementos aparecen «en una cadena sintáctica inmediatamente seguidos de un nombre o de un sintagma nominal comparten el rasgo $[+\mathrm{N}]$; mientras que nombres y adjetivos forman una clase sobre la base del rasgo en común [-N], por lo que no poseen el comportamiento distribucional mencionado» (Fernández y Anula 1995: 89). Los rasgos categoriales que los autores mencionados asignan a las piezas léxicas son los siguientes:

\section{Sistemas de rasgos categoriales}

\begin{tabular}{|l|c|c|}
\hline \multirow{2}{*}{ Clases de palabras } & \multicolumn{2}{c|}{ Tipos de rasgos } \\
\cline { 2 - 3 } & Nominal & Verbal \\
\hline Adjetivo & + & + \\
\hline Nombre & + & - \\
\hline Verbo & - & + \\
\hline Preposición & - & - \\
\hline
\end{tabular}

2 La información acerca de la TPP la hemos extraído sustancialmente del texto Sintaxis y Cognición. Introducción al conocimiento, el procesamiento y los déficits sintácticos de Marina Fernández Lagunilla y Alberto Anula Rebollo (1995). 
De manera particular nos interesan los rasgos propios de verbos y nombres; en primer lugar, porque el quechua no presenta preposiciones, y, además, porque tales propiedades categoriales son las involucradas en el cambio categorial que entraña el morfema «nominalizador». Si tuviésemos que describir ciertas entradas léxicas mediante estos rasgos sería de la siguiente forma:

puñu 'dormir':/puJu-/ (rasgos fonológicos)

$$
[+\mathrm{V},-\mathrm{N}] \quad \text { (rasgos categoriales) }
$$

wasi 'casa': /wasi-/(rasgos fonológicos)

$$
[-\mathrm{V},+\mathrm{N}] \quad \text { (rasgos categoriales) }
$$

Dado que el léxico presenta unidades o piezas que, de acuerdo con ciertas condiciones, se proyectan sintácticamente, es en este nivel que los procesos morfológicos operan, ya que la sintaxis no se ocupa del procesamiento de la información morfológica. Por consiguiente, la derivación, en tanto que tal, ocurre en el nivel léxico. La conformación de nuevas piezas léxicas que sustituyen sus rasgos categoriales es posible de acuerdo con los procesos intra- o transcategoriales que hemos definido en el $\S 1.1$., y es justo este procedimiento derivacional el que permite que el verbo puñu- 'dormir', al añadírsele el morfema derivativo -na, cambie a nombre. La modificación categorial sería la siguiente:

$$
\begin{array}{lll}
\text { /puJu-/ } & {[+\mathrm{V},-\mathrm{N}]} & \text { 'dormir' } \\
\text { /puJu-na/ } & {[-\mathrm{V},+\mathrm{N}]} & \text { 'lugar para dormir' }
\end{array}
$$

Respecto del sufijo $\{$-y $\}$, al ser un nominalizador de verbos, modificaría los rasgos de las bases verbales a través del mecanismo derivativo. Para ejemplificar este proceso presentaremos a continuación la reformulación del verbo llamka - 'trabajar' al interactuar con el sufijo «infinitivizador».

$$
\begin{array}{lc}
/ \lambda \text { amka- } / \rightarrow / \lambda \text { amka }-\mathrm{y} / & \text { (formas fonológicas) } \\
{[[\mathrm{X}] \mathrm{V}+\mathrm{y}] \mathrm{n} \quad \mathrm{V} \rightarrow \mathrm{N}} & \text { (regla derivativa) } \\
{[-\mathrm{N},+\mathrm{V}] \rightarrow[+\mathrm{N},-\mathrm{V}]} & \text { (modificación de rasgos categoriales) }
\end{array}
$$

Notamos que el sufijo determina el cambio de información categorial, necesario para que este conforme el repertorio de sufijos nominalizadores deverbativos. Además de los rasgos categoriales es menester señalar que, en lo que concierne a las piezas verbales, estas definen una estructura argumental distinta dependiendo de los morfemas derivativos. De esta manera, verbos que son intransitivos y que, por lo tanto, requieren un solo argumento, pasan a ser transitivos, es decir, su red argumental define dos posiciones que deben ser ocupadas por argumentos de tipo nominal (Rocha 2008: 467). Al referirnos 
a estructuras argumentales de tipo nominal no nos referimos necesariamente a núcleos nominales, ya que puede ser una estructura subordinada cumpliendo tal función.

La derivación como proceso de formación de vocabulario nuevo tiene un estatus léxico. Una de las asunciones básicas de esta propuesta es que el componente sintáctico no procesa la información morfológica, lo cual, además, implica la determinación de un hiato en lo que concierne a los mecanismos que afectan a la sintaxis de aquellos que involucran a las formas lexicales (Marantz 1998).

No obstante, el problema se suscita en términos de la asignación de caso que las formas nominalizadas le confieren a elementos oracionales de tipo nominal. Esto es, las nuevas formas nominales definen argumentos internos en construcciones complejas, los cuales portan la marca de caso acusativo - $t a$. Esto teóricamente es improbable, debido a que los elementos verbales son los únicos capaces de asignar caso acusativo a elementos nominales. Veamos la siguiente oración propuesta por Parker (1965: 58):

(i) /čakrata $\lambda$ amkayta munani/ 'quiero trabajar la chacra'

En la oración anterior el verbo principal es el verbo muna- 'querer', el cual define una estructura argumental compleja formada por la estructura chakrata llamkayta 'trabajar la chacra'. El verbo principal le asigna caso acusativo al verbo subordinado llamka-y; por esa razón en el ejemplo aparece la forma -ta al final de este. Sin embargo, la pregunta que se desprende es qué elemento le asigna caso acusativo al elemento nominal chakra. No sería conducente que sea el verbo muna- el asignador, puesto que tanta 'pan' es el argumento definido por miku- 'comer', y se encuentra en un dominio distinto. El mismo escollo lo encontramos en las siguientes oraciones:

(ii) Nuqa tanta -ta ranti $-\mathrm{y}-$ ta muna: Yo Pan ACUS. Comprar INF ACUS querer 1PA

'Quiero comprar pan'

(iii) Ñuqa yaku -ta upya $-\mathrm{y}-$ ta muna:

Yo agua ACUS. Tomar INF ACUS querer 1PA

'Quiero tomar agua'

Tanto en (ii) como en (iii) notamos que las raíces nominales tanta 'pan' y yaku 'agua' portan la marca de acusativo; en ambos casos, plantear que es el verbo muna- 'querer' el que asigna caso es inconsistente por cuanto ambos son argumentos definidos por los pares léxicos ranti- 'comprar' y upya- 'tomar'; en consecuencia, estos últimos deberían proyectarse desde sintagmas verbales. Para que esto ocurra, las formas señaladas, cuyo morfema es el infinitivizador $\{-\mathrm{y}\}$, y que hacen posible tal proyección, deben ser categorialmente verbos. 
Respecto de la asignación de caso, el quechua cuenta con morfemas específicos para definir que un argumento porte tal marca. Los únicos elementos léxicos que pueden asignar caso, teóricamente, son aquellos que carecen de propiedades nominales:

«Puesto que los SSNN son los receptores del caso, ahora debemos precisar qué elementos son los asignadores de caso [...] la propiedad de asignar Caso está relacionada con el rasgo categorial [-N] que poseen en común V y P, y del que carecen N y A» (Fernández y Rebollo 1997: 167).

La respuesta más adecuada que permite explicar la naturaleza de las formas verbales devenidas en nombres es que el morfema en cuestión permite la correncia de verbos sin referencia de persona ni tiempo; esto es, no es una forma nominal propiamente, sino una forma verbal impersonal. No es raro encontrar formas verbales impersonales que cumplan funciones nominales. Tal es el caso del español, cuyos verbos en su forma infinitiva cumplen funciones nominales en la oración sin ningún problema.

(iv) Fumar es excesivamente dañino.

En la oración anterior, notamos que la forma infinitiva fumar ocupa el lugar que de manera canónica le corresponde a una estructura de tipo nominal; no obstante, sería impreciso asumir que se trata de un nombre, ya que las propiedades categoriales de la pieza léxica se mantiene incólume. En lo que concierne a la estructuración, tendríamos que plantear que esta pieza verbal exigida por el copulativo ser proyecta su propio sintagma verbal, de modo que esta forma verbal impersonal es una oración subordinada incrustada, razón por la cual es menester proponer la preservación de los rasgos idiosincrásicos de una raíz verbal $[+\mathrm{V},-\mathrm{N}]$.

La naturaleza impersonal de los verbos cuya terminación es -y es más adecuada con el nombre que se le da a este morfema: derivativo infinitivizador. Además, siguiendo este razonamiento, encontramos una explicación plausible respecto de la asignación de la marca de acusativo al núcleo nominal chakra, a través del verbo llamkay (obviando, por supuesto, la propuesta que lo concibe como producto de un proceso de nominalización). Los marcadores de frase que representan los dominios sintácticos de los elementos lexicales cuyos rasgos son $[+\mathrm{V},-\mathrm{N}]$, a saber, mikuy y llamkay, tienen que determinar estructuralmente el dominio verbal en el que se encuentran. Para efectos del caso, presentamos el siguiente esquema, siguiendo los lineamientos formales de la teoría X barra, para ejemplificar la oración (ii) ${ }^{3}$ :

3 El esquema arbóreo se realizó de acuerdo con la disposición que permite el programa virtual denominado phpSyntaxTre cuyo enlace, revisado el 23 de octubre de 2010 es http://ironcreek. net/phpsyntaxtree/?.

LETRAS 81 (116), 2010 


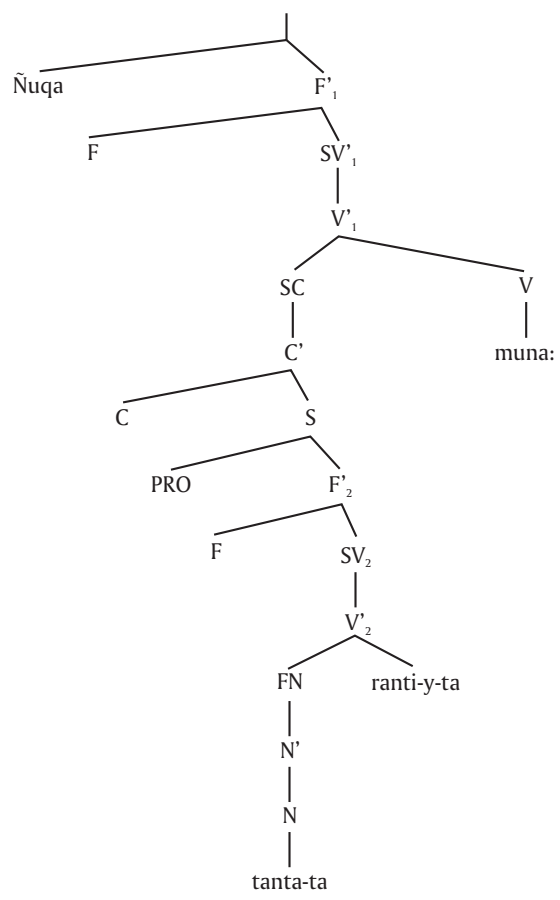

Una evidencia insoslayable a favor de la propuesta del infinitivizador como marca propia de verbos impersonales es la que encontramos en las variedades quechuas de Aurahuá y Chupamarca (Huancavelica), y en la variedad de Huánuco (Huamalíes). En las poblaciones jóvenes existe un uso generalizado de las formas infinitivas del español para referirse a verbos descontextualizados y en su forma impersonal. Así tenemos que el prototípico miku-y es para los nativohablantes de estas comunidades miku-r, el verbo muna-y es muna-r, y así por ende. Asumimos que, dada la polifuncionalidad del morfema en cuestión, la forma más simple de verbalizar una forma impersonal es a través de la terminación castellana. Además, los quechuahablantes bilingüies realizan el desplazamiento de estas propiedades debido a la correspondencia que existe entre la función infinitiva de la terminación quechua $\{-\mathrm{y}\}$ y el infinitivo castellano. Una evidencia que respalda nuestro aserto es que los verbos infinitivos del castellano pueden aparecer como formas nominales propiamente dichas, e incluso portar los elementos formales que acompañan al nombre: artículos, adjetivos, etc. (RAE Manual 2010: 494). Así tenemos formas como las siguientes:

(v) El bajar la cabeza ante la autoridad me parece indigno. 
(vi) El inefable retumbar de las olas me abstrae.

Tanto el verbo bajar como el verbo retumbar aparecen como núcleos nominales en las estructuras de Sujeto (S), de manera que las propiedades idiosincrásicas de estos elementos nominales son de naturaleza sintáctica. La asignación de la terminación impersonal del castellano $-r$ no es gratuita ni casual, se sustenta en la similitud con la del quechua.

No hemos detectado evidencia que constate una predicción a partir del análisis dado; esto es, no existe evidencia de oraciones estructuradas de la siguiente manera:

(vii) Nuqa tanta-ta miku-r-ta munani.** 'quiero comer pan'

(viii) Asunta yaku-ta upya-r-ta munan.* 'quiero tomar agua'

En los datos hipotéticos presentados en (vii) y (viii) vemos que las formas infinitivas quechuas con la terminación castellana en construcciones complejas sería agramatical. A pesar de ello, el proceso de reemplazo en ciertas variedades del quechua se hace evidente en formas descontextualizadas de una estructura sintáctica.

En suma, nuestro objetivo sustancial ha sido presentar las debilidades de la categorización del morfema infinitivizador como una maraca denominativa, esto es, como una marca que deriva nombres a partir de verbos. Asumimos que una propuesta más acorde con la evidencia que nos ofrece el quechua sostiene que esta marca, tal y como su propio nombre indica, define formas verbales impersonales.

\section{Conclusiones}

1. El quechua presenta un sufijo universal que ha sido clasificado como derivativo nominalizador, que modifica raíces verbales y las vuelve verbos. A esta marca morfológica se le ha denominado tradicionalmente «infinitivizador».

2. La denominación es imprecisa por cuanto una marca infinitiva no implica de manera inconcusa que la raíz verbal a la que se añade el morfema cambie sus propiedades categoriales.

3. Las propiedades categoriales de una raíz verbal son $[+\mathrm{V},-\mathrm{N}]$. Si tales rasgos cambian al par $[-\mathrm{V},+\mathrm{N}]$, implica que los verbos devienen en nombres $\mathrm{y}$, en consecuencia, pierden sus propiedades sintácticas de definir argumentos y de ser propiamente asignador de caso.

Letras 81 (116), 2010 
4. En las construcciones subordinadas vemos que los argumentos definidos por las formas verbales «nominalizadas» reciben caso acusativo y, por lo tanto, las propiedades verbales no se pierden. Esta evidencia supone que la marca infinitiva no es propiamente un nominalizador.

5. Una evidencia concreta de que la marca infinitiva no es un derivativo nominal es que en ciertas variedades quechuas como las de Aurahuá-Chupamarca y la de Huánuco, se está adaptando la terminación castellana $\{-r\}$ para denominar formas verbales descontextualizadas; a saber, fuera de toda estructura sintáctica.

6. El marcador de frase permite evaluar los dominios sintácticos de las formas verbales involucradas. Planteamos, en consecuencia, que el morfema quechua es efectivamente una marca infinitiva, entendida como una maraca que acompaña a formas verbales sin referencia de tiempo y persona.

\section{Referencias bibliográficas}

CERRÓN-PALOMINO, Rodolfo (1987). Lingüística quechua. Cusco, Centro de estudios rurales andinos «Bartolomé de Las Casas».

CERRÓN-PALOMINO, Rodolfo (1994). Quechumara. Estructuras paralelas de las lenguas quechua y aimara. La Paz, Centro de Investigación y Promoción del campesinado.

CERRÓN-PALOMINO, Rodolfo (1994). Quechua sureño. Diccionario unificado. Lima, Biblioteca Básica Peruana.

FERNÁNDEZ L., Marina y Alberto ANULA (1995). Sintaxis y cognición. Madrid: Editorial Síntesis.

LEFEBVRE, Claire y Pieter MUYSKEN (1988). Mixed Categories. Nominalizations in Quechua. Dortrecht, Boston y London, Kluwer Academic Publishers.

LIRA, Jorge (1941). Diccionario Kkechuwa-español. Tucumán, Universidad Nacional de Tucumán (Departamento de Investigaciones Regionales).

MARANTZ, Alec (1988). «No Escape From Syntax: Don't Try Morphological Analysis in the Privacy of Your Own Lexicon», in «Proceedings of the 1998 Penn Linguistics Colloqium», ed. by Alexis Dimitriadis, available from Penn Working. Consultado el 30 de octubre de 2010. < http://dingo.sbs.arizona.edu/ hharley/courses/Oxford/Marantz.pdf>

PARKER, Gary (1965). Gramática del quechua de Ayacucho. Lima, Universidad Nacional Mayor de San Marcos.

PARKER, Gary (1973). Derivación verbal en el quechua de Ancash. Lima, CILA, Documento de trabajo N..$^{\circ} 25$.

PARKER, Gary (1976). Gramática quechua: Ancash-Huailas. Lima, Ministerio de Educación e Instituto de Estudios Peruanos.

PARKER, Gary y Amancio Chávez (1976). Diccionario Quechua: Ancash-Huaylas. Lima, Ministerio de Educación e I.E.P.

QUESADA CASTILLO, Félix (1976). Gramática quechua: Cajamarca-Cañaris. Lima, Ministerio de Educación e Instituto de Estudios Peruanos. 
REAL ACADEMIA ESPAÑOLA (2010). Manual de la Nueva Gramática de la Lengua Española. Madrid, Espasa Libros.

ROCHA, Rolando (2007). «Derivación verbal en quechua e hipótesis lexicalista». En: Marco Martos, Aída Mendoza e Ismael Pinto Vargas (eds.) Actas del II Congreso de Lexicología y Lexicografía «Pedro Benvenuto Murrieta». Lima, Academia Peruana de la Lengua y Universidad de San Martín de Porres; pp. 463-476.

SANTO TOMÁS, Domingo de (1951). Lexicón o vocabulario de la lengua general del Perú. Lima, Edición Facsimilar publicada con prólogo de Raúl Porras Barrenechea.

SCALISE, Sergio (1987). Morfología generativa. Madrid, Alianza Editorial.

TORERO, Alfredo (1964). El quechua y la historia social andina. Lima, Universidad Ricardo Palma.

TORERO, Alfredo (2000). «El marco histórico geográfico en la interacción quechua-aru». En Escritura y Pensamiento, 5; pp. 9-58.

TORERO, Alfredo (2002). Idiomas de los Andes. Lingüística e Historia. Lima, Editorial Horizonte e IFEA.

SIN AUTOR (1905). Vocabulario políglota incaico. Lima, Tipografía del Colegio de Propaganda Fide del Perú. 